\title{
Human immunodeficiency-causing mutation defines CD16 in spontaneous NK cell cytotoxicity
}

\author{
Jennifer T. Grier, ${ }^{1}$ Lisa R. Forbes, ${ }^{1}$ Linda Monaco-Shawver, ${ }^{1}$ Jennifer Oshinsky, ${ }^{2}$ \\ T. Prescott Atkinson, ${ }^{3}$ Curtis Moody, ${ }^{4}$ Rahul Pandey, ${ }^{1}$ Kerry S. Campbell, ${ }^{2}$ and Jordan S. Orange ${ }^{5}$ \\ ${ }^{1}$ Children's Hospital of Philadelphia Research Institute, Philadelphia, Pennsylvania, USA. ${ }^{2}$ Fox Chase Cancer Center, Philadelphia, Pennsylvania, USA. \\ 3University of Alabama Birmingham, Birmingham, Alabama, USA. ${ }^{3}$ Boston Children's Hospital, Boston, Massachusetts, USA. \\ ${ }^{5}$ Baylor College of Medicine, Texas Children's Hospital, Houston, Texas, USA.
}

\begin{abstract}
The Fc receptor on NK cells, Fc $\gamma$ RIIIA (CD16), has been extensively studied for its role in mediating antibodydependent cellular cytotoxicity (ADCC). A homozygous missense mutation in CD16 (encoding a L66H substitution) is associated with severe herpesvirus infections in rare patients. Here, we identified a new patient with this CD16 mutation and compared the patient's NK cells to those of the originally reported patient. Patients with the L66H mutation had intact ADCC, but deficient spontaneous NK cell cytotoxicity and decreased surface expression of CD2, a coactivation receptor. Mechanistic studies in a human NK cell line, NK-92, demonstrated that CD16 expression correlated with CD2 surface levels and enabled killing of a melanoma cell line typically resistant to CD16-deficient NK-92 cells. An association between CD16 and CD2 was identified biochemically and at the immunological synapse, which elicited CD16 signaling after CD2 engagement. Stable expression of CD16 L66H in NK-92 cells recapitulated the patient phenotype, abrogating association of CD16 with CD2 as well as CD16 signaling after CD2 ligation. Thus, CD16 serves a role in NK cell-mediated spontaneous cytotoxicity through a specific association with CD2 and represents a potential mechanism underlying a human congenital immunodeficiency.
\end{abstract}

\section{Introduction}

NK cells are innate lymphocytes important in host defense. They participate in defense against infections and immune surveillance of cancerous cells $(1,2)$. A major function of NK cells is that of cytotoxicity, initiated following the ligation of germline-encoded receptors by ligands on target cells (3). NK cell cytotoxicity is contact dependent and requires the formation of a specialized immunological synapse with a target cell, through which the contents of lytic granules are secreted (4). Cytotoxicity is induced when the balance of activation signaling achieves a threshold. This can be achieved after recognition of target cells, either expressing sufficient ligands for NK cell activation receptors or opsonized with IgG, leading to antibody-dependent cellular cytotoxicity (ADCC). The NK cell activating receptor responsible for recognizing IgG and enabling ADCC is Fc $\gamma$ RIIIA (also known as CD16; refs. 2, 5).

CD16 is a low-affinity receptor for IgG Fc expressed on phagocytes and NK cells (6). CD16A, the form expressed on NK cells, has 2 extracellular Ig domains $(7,8)$, a short cytoplasmic tail, and a transmembrane domain that enables its association with the immunoreceptor tyrosine-based activation motif-containing adaptors TCR $\zeta$ and Fc- $\varepsilon$ RI- $\gamma(9)$. IgG binds to CD 16 via its second, membrane-proximal Ig domain, which can be recognized by the mAb 3 G8 (10) and promotes TCR $\zeta$ phosphorylation and signal transduction (11). The first, distal Ig domain of CD16 can be recognized by mAb B73.1 (12), although function of this domain is poorly understood (8).

Conflict of interest: CD16.NK-92 cells are patented for use by Kerry S. Campbell and licensed by Conkwest.

Citation for this article: J Clin Invest. 2012;122(10):3769-3780. doi:10.1172/JCI64837.
NK cell function is essential in human host defense. This is demonstrated by the susceptibility of patients lacking or having functionally deficient NK cells to infections with herpesvirus and human papilloma virus (HPV) $(13,14)$. Human mutation of CD16A has been classified as a primary immunodeficiency and functional NK cell deficiency (15). It was the first human congenital single-gene abnormality identified as having an isolated effect on NK cell function. Specifically, a homozygous $\mathrm{T}$ to A missense substitution at position 230 in the FCGR3A gene, resulting in a $\mathrm{L}$ to $\mathrm{H}$ alteration at position 66 in the first Ig-like domain of CD16 (referred to herein as the L66H mutation), was identified in 2 separate reports as being associated with functional NK cell deficiency $(16,17)$. Homozygosity of this variant is likely rare in diverse populations, based on the limited number of these sequences in genome databases, such as International HapMap (http://www.hapmap.org), Thousand Genomes (http:// www.1000genomes.org), and NHLBI Exome Sequencing Project (http://evs.gs.washington.edu). A 5-year-old girl homozygous for this mutation had frequent upper respiratory infections, recurrent herpes simplex virus (HSV) stomatitis, and recurrent herpes whitlow (16). A homozygously affected 3-year-old male had recurrent upper respiratory infections, prolonged EBV (also known as Castleman's disease), recurrent cutaneous HSV, and varicella zoster virus infections (17). Interestingly, the CD16 alteration in these patients did not prevent receptor expression, but abrogated its recognition by $\mathrm{mAb}$ B73.1. Although the female patient had defective NK cell spontaneous cytotoxicity, ADCC was curiously intact in both patients $(16,17)$, which suggests that the immunodeficiency did not result from an impaired ability of the mutant receptor to interact with IgG. In contrast, a common polymorphism in the membrane-proximal Ig domain, resulting in either 
A
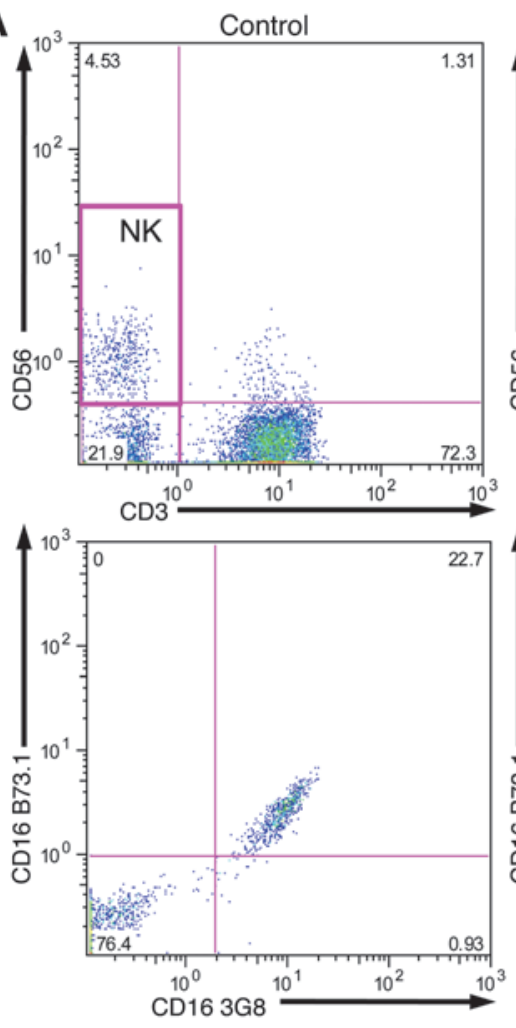

B
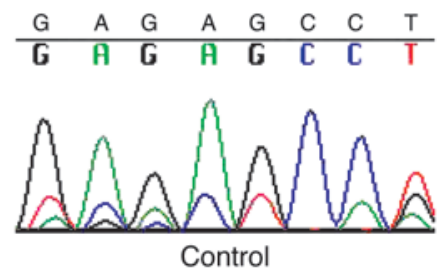
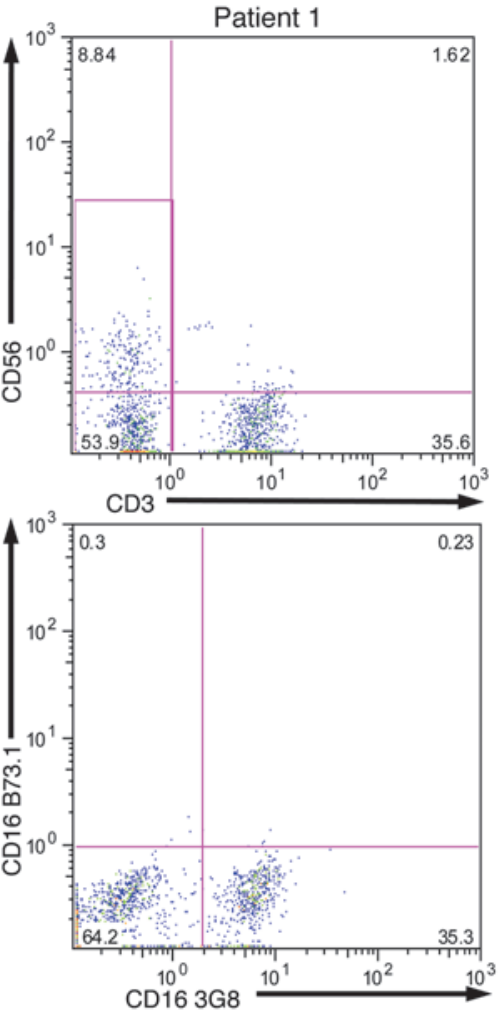

C

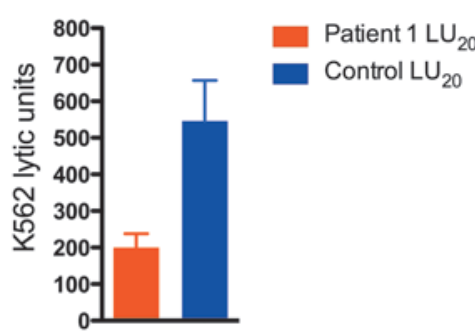

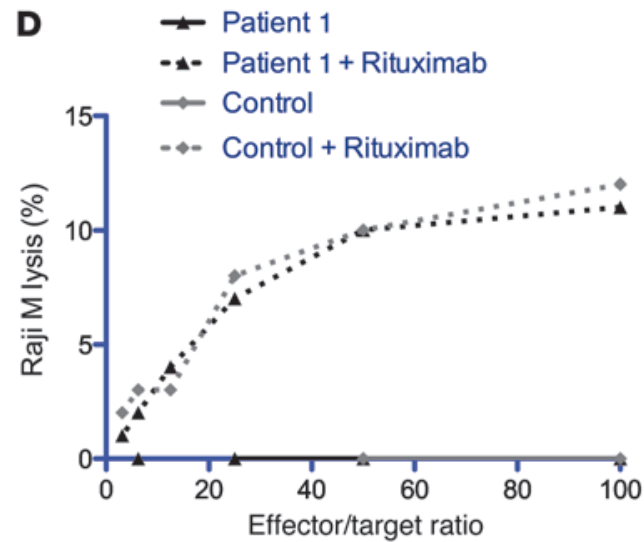

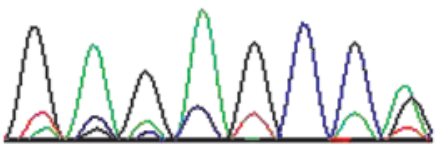

Patient 1

Figure 1

Point mutation in CD16 results in CD16 B73.1 epitope loss and decreased natural killing without affecting ADCC. (A) FACS analysis of CD3CD56+ NK cells from a control donor and patient 1 for CD16 3G8 and CD16 B73.1 epitopes. (B) Sequence analysis of CD16 within the region corresponding to the B73.1 epitope in a normal donor and patient 1. (C) NK cell lytic units $\left(\mathrm{LU}_{20}\right)$ against K562 cells for patient 1 and control ( $n=3$ independent experiments). (D) ADCC assay of patient 1 or control PBMCs against Raji cells in the presence or absence of rituximab (representative of $n=3$ independent experiments).

$\mathrm{V}$ or $\mathrm{F}$ at position 176 , contributes to higher or lower affinity, respectively, toward IgG Fc. Thus, the $\mathrm{L} 66 \mathrm{H}$ mutation in the first Ig-like domain markedly affects spontaneous cytotoxicity, whereas the $\mathrm{V} 176 \mathrm{~F}$ polymorphic variation in the second Ig-like domain substantially influences the strength of ADCC responses by NK cells. These natural sequence alterations indicate that the 2 Ig-like domains govern distinct functions.

Here, we identified a new patient homozygous for FCGR3A mutation at position 230, a 14-year-old male with recurrent EBVassociated Castleman's disease and HPV infection (patient 1; see Methods). As with prior patients, he did not possess the mAb B73.1-recognized CD16 epitope and had deficient NK cell cytotoxicity, but preserved ADCC. Since the CD16 defect in these patients does not abrogate ADCC, we investigated the molecular mechanism by which CD16 participates in spontaneous cytotoxicity by NK cells. We found that CD16 clustered at the lytic immunological synapse, as well as increased the surface expres- sion of and associated with the CD2 coactivating receptor to enhance NK cell activation after CD2 ligand binding. This implicates a costimulatory role for CD16 in NK cell cytotoxicity independent of IgG Fc binding, which is a vital function in human host defense.

\section{Results}

CD16 mutation and loss of B73.1 epitope does not impair ADCC, but decreases NK cell spontaneous cytotoxicity. The unusual manifestation of EBV and HPV in patient 1 (Supplemental Figure 1; supplemental material available online with this article; doi:10.1172/ JCI64837DS1) with an otherwise normal clinical immunologic assessment prompted evaluation of his NK cells. His peripheral blood percentage of $\mathrm{CD}^{2} 6^{+} \mathrm{CD} 3^{-} \mathrm{NK}$ cells was within normal agespecific ranges (18). Both patient 1 and control NK cells were recognized by anti-CD16 mAb 3G8, demonstrating the presence of CD16 on their surface, but only control NK cells were recognized 
A

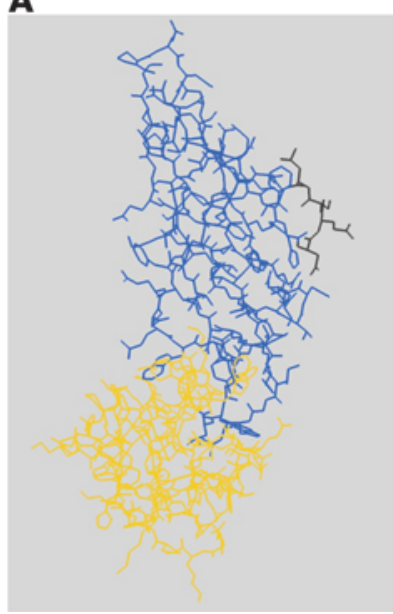

B

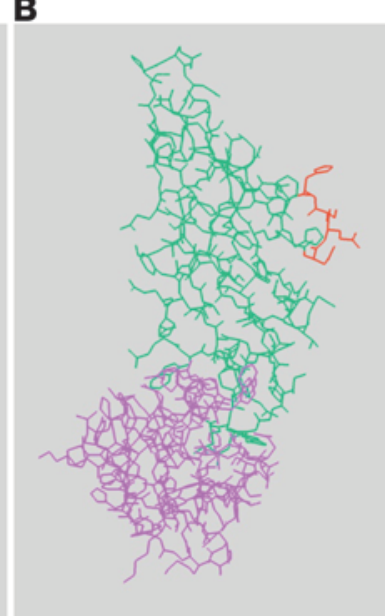

C

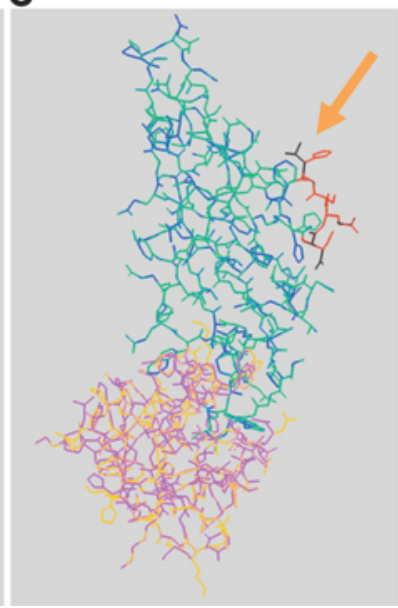

\section{Figure 2}

Model of mutant CD16 structure. (A) Rendering of the published wild-type CD16 structure. Yellow, membrane-proximal Iglike domain; blue, membrane-distal Ig-like domain; black, B73.1 epitope loop. (B) Predicted CD16 L66H mutant structure. Purple, proximal domain; green, membrane-distal domain; red, B73.1 epitope loop. (C) Overlay of wild-type and $\mathrm{L} 66 \mathrm{H}$ CD16 structures. Orange arrow denotes L66H abnormality. by anti-CD16 B73.1 mAb (Figure 1A), demonstrating the loss of the specific epitope in patient 1 . To determine whether this loss was associated with genetic alteration of the corresponding CD16 gene region, sequence analysis was performed. A nucleotide substitution of $\mathrm{T}$ to $\mathrm{A}$ at position 230 was present on both alleles (Figure 1B). This predicts the $\mathrm{L} 66 \mathrm{H}$ mutation in the first extracellular domain, which was previously reported to disrupt the B73.1, but not the 3G8, epitope (16).

To determine the effect of the patient mutation and CD16 alteration upon NK cell function, cytotoxicity was evaluated. PBMCs of patient 1 had an almost 3-fold reduction of K562 target cell killing (Figure 1C). In contrast, ADCC against Raji target cells was retained and comparable to that of control cells (Figure 1D). Thus, like the original patient (referred to herein as patient 2; ref. 16), patient 1 had unusual susceptibility to severe viral infections that correlated with CD16 L66H mutation, loss of mAb B73.1 binding, and decreased NK cell cytotoxicity with intact ADCC.

We next asked how this CD16 mutation could affect the receptor. Given the loss of B73.1 mAb epitope, we hypothesized that $\mathrm{L} 66 \mathrm{H}$ mutations alter the 3D structure of CD16. Since the crystal structure of the extracellular domain of wild-type CD16 has been solved to $3.2 \AA$, we used the $3.2 \AA$ structure of wild-type CD16 (Figure 2A and ref. 19) and modeled the L66H mutant, which predicted a shift in the B73.1 epitope loop as well as numerous small side chain orientations (Figure 2B). An overlay demonstrated that the overall structure of the mutant protein was predicted to be intact, save the aforementioned small alterations (Figure 2C). This supported the observation that $\mathrm{ADCC}$ requiring $\mathrm{Fc}$ binding did not affect patient cells and provided a structural explanation for B73.1 epitope loss.

Surface expression of CD2 is altered in patients with CD16 L66H mutation. Although patient cells expressing CD16 L66H mutations had decreased NK cell cytotoxicity, it seemed unlikely that this was caused by CD16 acting directly as a triggering receptor for NK cell cytotoxicity. We therefore evaluated NK cells of patient 1 in detail via flow cytometry to determine whether the homozygous mutation caused an additional phenotypic effect (Figure 3A). In multiple blood samples obtained over 2 years, a significant decrease in CD2 surface expression on patient 1 NK cells was noted (Figure 3B). With the exceptions of CD16 B73.1 and NKG2D, other phenotypic differences between patient 1 and control NK cells were not significant. Within the minor, developmentally immature CD56 ${ }^{\text {bright }}$ NK cell subset, few cells express surface CD16 (20). Thus, in order to consider a potential role for the mutant CD16 in surface expression of CD2, peripheral blood CD56 $6^{\text {bright }}$ and CD56 $6^{\mathrm{dim}} \mathrm{NK}$ cell subsets were evaluated separately. The decrease in CD2 surface expression on cells of patient 1 was limited to CD56 $6^{\mathrm{dim}} \mathrm{NK}$ cells, which were primarily CD $16^{+}$(Supplemental Figure 2, A and B), as CD2 expression on CD16-CD56 $6^{\text {bright }} \mathrm{NK}$ cells was equivalent in patient 1 and the control (Supplemental Figure 2C). This raises the possibility that CD16 participates in optimal surface expression of CD2 in mature NK cells.

To confirm the correlation between homozygous CD16 L66H mutation and CD2 surface expression observed in patient 1, we obtained blood from patient 2 , the patient originally reported as being affected by this mutation (16). The NK cell phenotype of patient 2 also demonstrated absent B73.1 epitope detection and decreased CD2 surface expression (Figure 3, C and D). Also similar to patient 1 , the CD2 decrease was only noted in CD56 dim (Supplemental Figure 2E), but not CD56 bright (Supplemental Figure 2F), NK cell subsets relative to control, albeit in a much smaller total percentage of NK cells (Supplemental Figure 2D). Although NKG2D appeared altered in patient 1, this trend was not observed in patient 2 . Thus, overall surface expression of CD2 in these patients appeared to be specifically negatively affected by

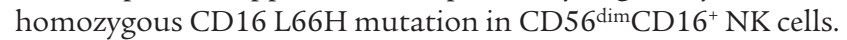

CD16-expressing NK-92 cells demonstrate a role for CD16 in cytotoxicity. Based on the patient observations, we wanted to determine if there could be a role for CD16 in NK cell cytotoxicity irrespective of ADCC. We used the immortalized human NK cell line NK-92, which does not endogenously express CD16, and compared it with a sub-line stably transduced to express CD16 (referred to herein as CD16.NK-92; ref. 21). To determine whether exogenous expression of CD16 affects the presence of NK cell surface receptors, the flow cytometry-based phenotypic analysis used above was applied to NK-92 and CD16.NK-92 cells (Figure 4A). Over multiple repetitions, the CD16.NK-92 cell line expressed a significantly greater amount of CD2 on the cell surface compared with the parental NK-92 line (Figure 4B). Thus, wild-type CD16 expression positively affected surface CD2 levels.

We next asked whether the presence of CD16 in this cell line could contribute to NK cell cytotoxicity. To validate the physi- 

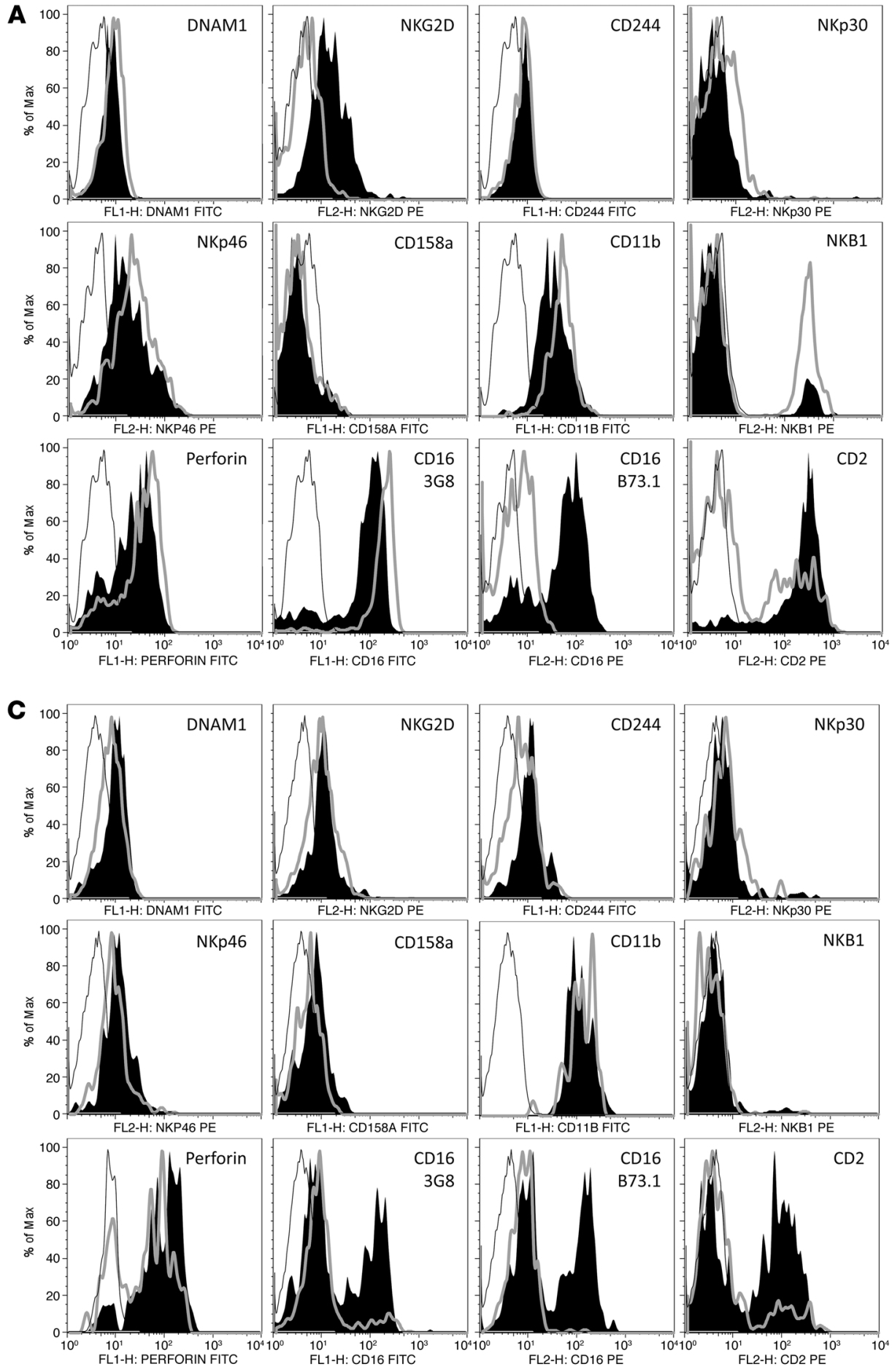

FL1-H: CD158A FITC
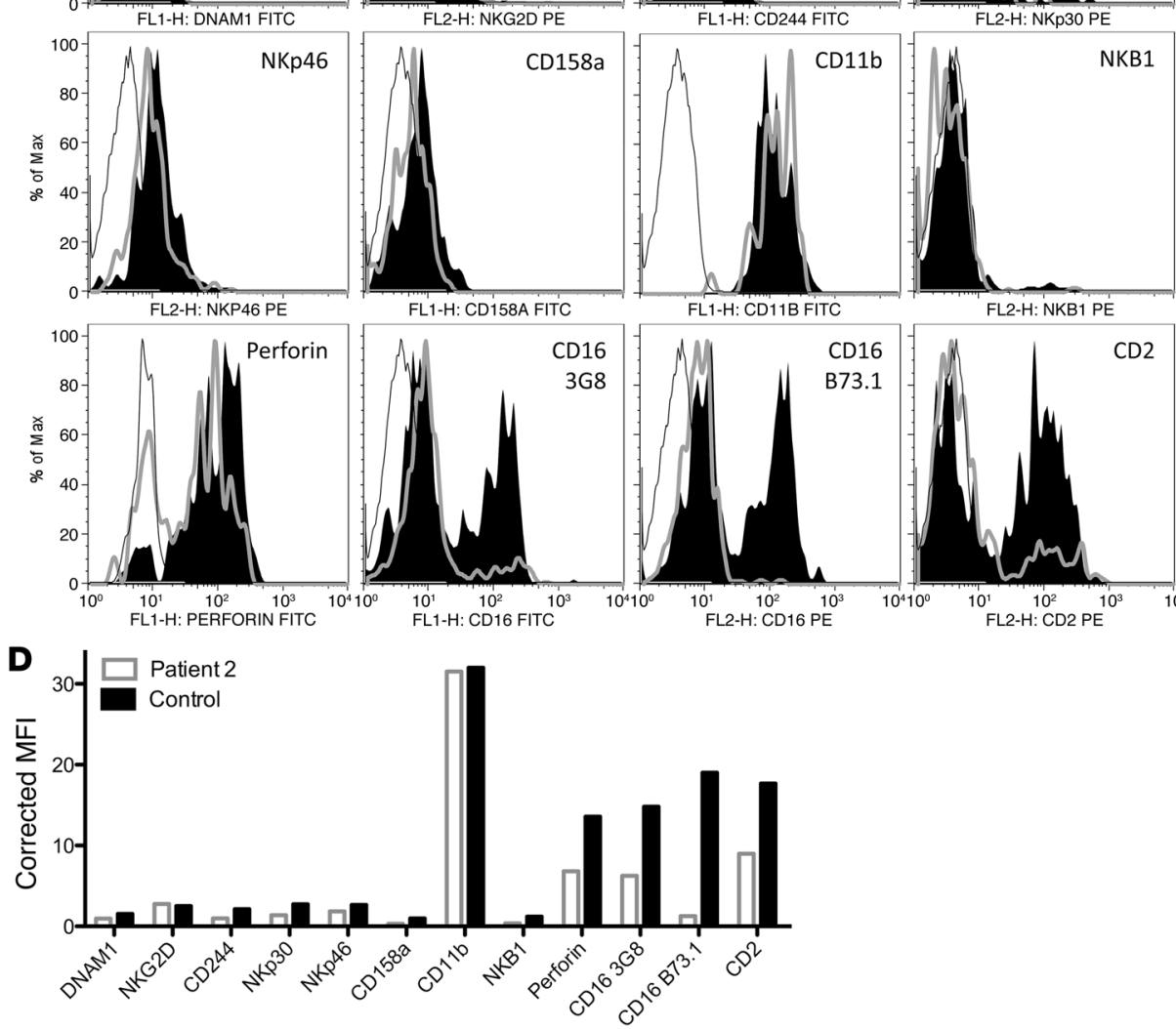
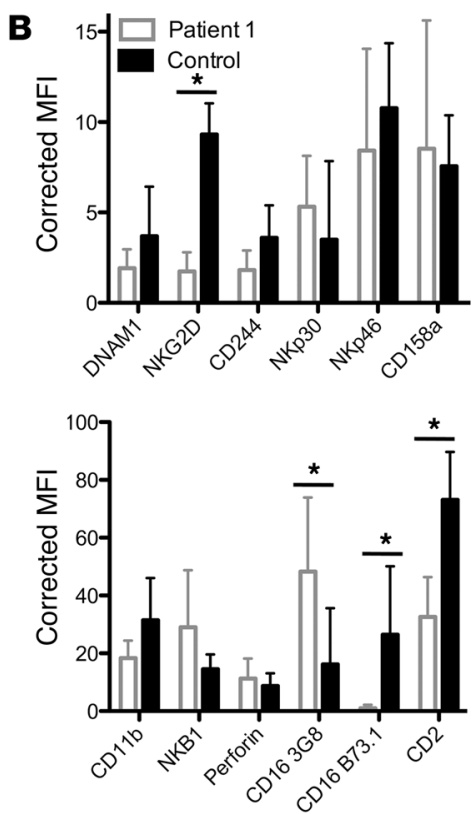

Figure 3

NK cell phenotype of $\mathrm{L} 66 \mathrm{H}$ patients. PBMCs were gated on CD3-CD56+ NK cells from patient 1 (A and $B$ ) or patient 2 (C and D). (A) FACS analysis for the indicated NK cell markers (thick gray line, patient 1; black filled regions, control donor; thin black line, isotype control), representative of 3 independent experiments from 3 independent blood draws. (B) Quantitative analysis of MFI for each marker relative to the isotype control. ${ }^{\star} P<0.05$, paired Student's $t$ test. (C) FACS analysis for the indicated NK cell markers (thick gray line, patient 2; black filled regions, control donor; thin black line, isotype control). Only 1 sample was available, and although a relatively small population of CD16 NK cells overall was noted, they were $3 G 8^{+} B 73.1^{-}$. (D) Quantitative analysis of MFI for each marker relative to the isotype control. 

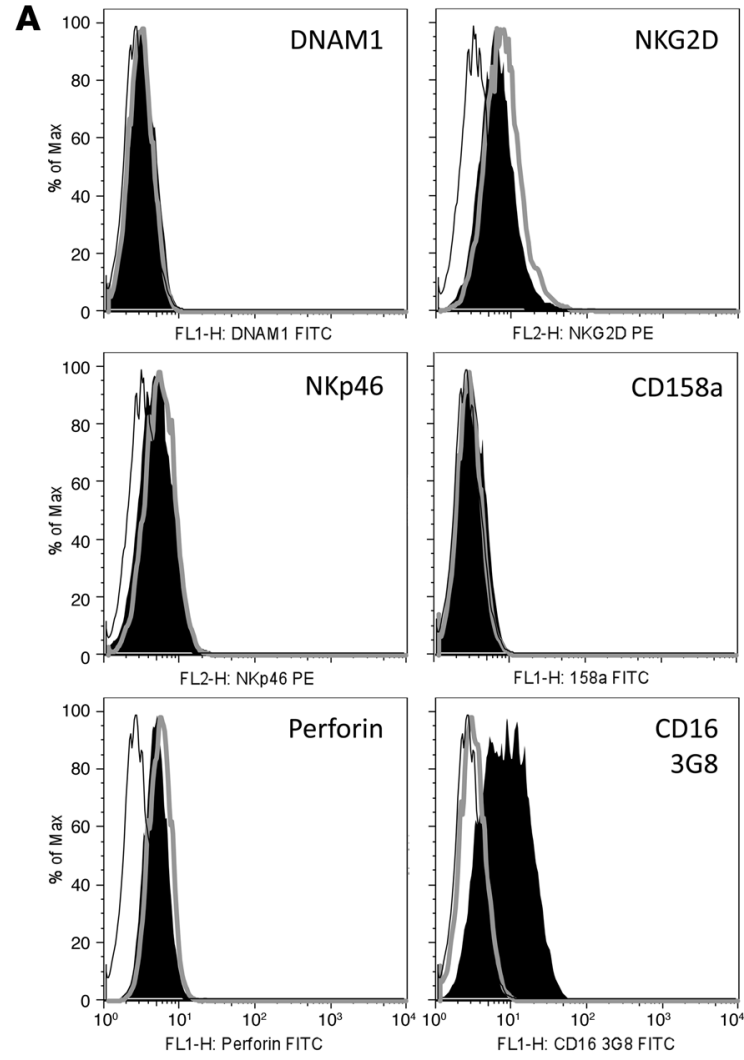

FL1-H: 158a FITC
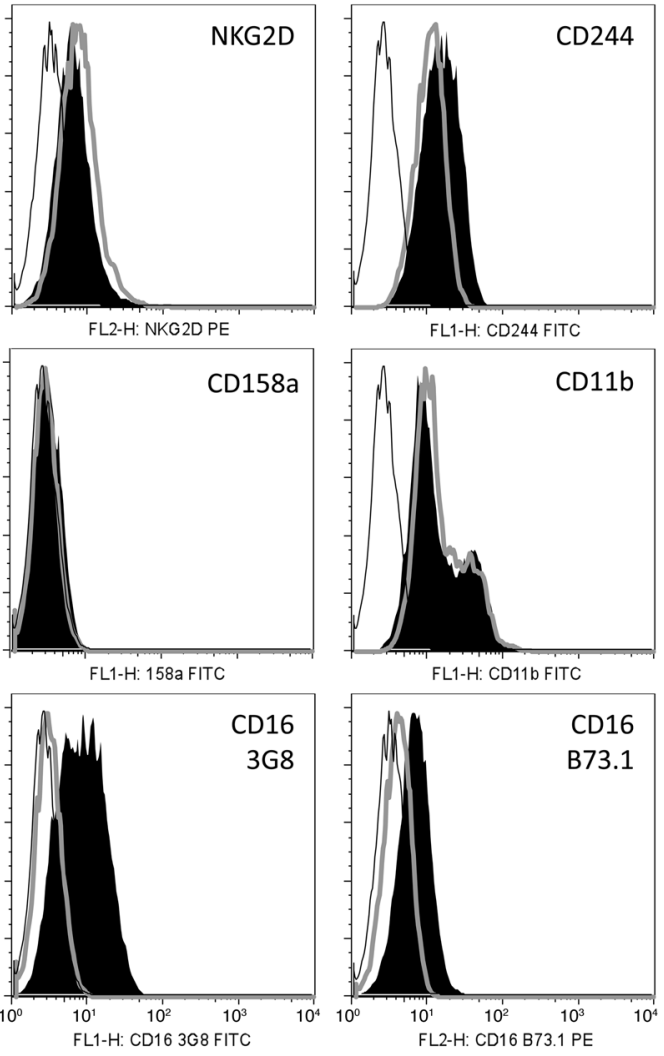

FL1-H: CD244 FITC

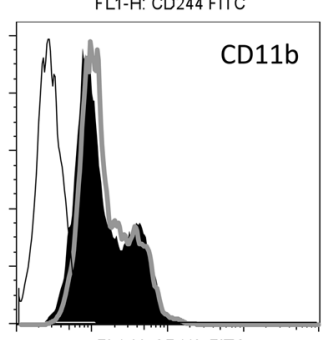

FL1-H: CD11b FITC
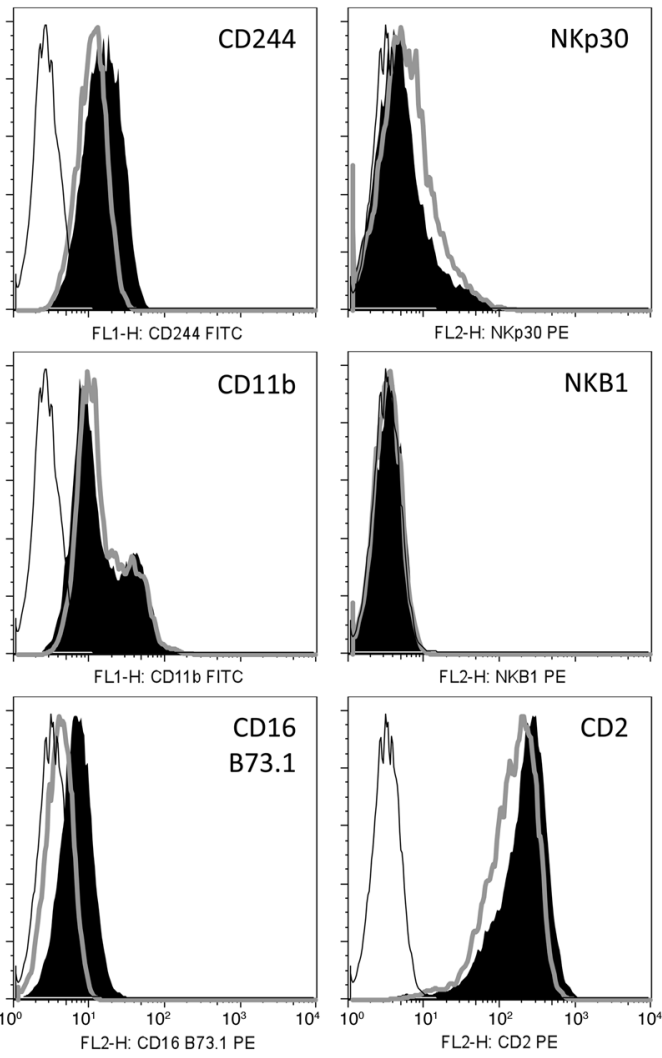

FL2-H: NKB1 PE

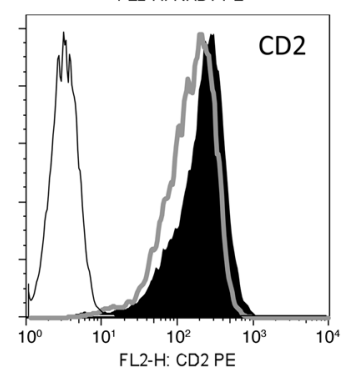

B

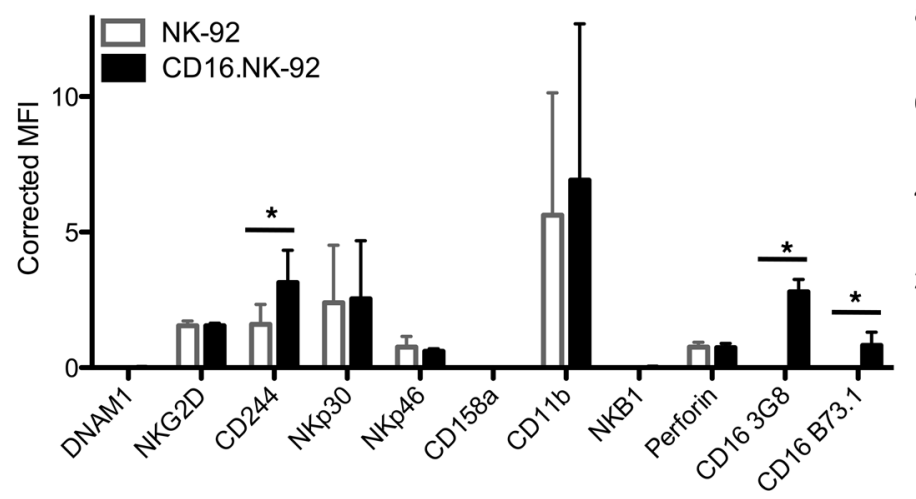

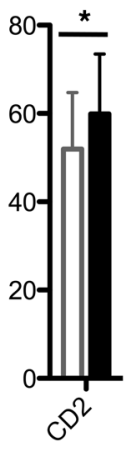

\section{Figure 4}

Phenotype of NK-92 and CD16.NK-92 NK cell lines. (A) FACS analysis of the indicated NK cell markers (thick gray line, NK-92; black filled regions, CD16.NK-92; thin black line, isotype control), representative of 3 independent experiments. (B) Quantitative analysis of MFI for each marker relative to isotype over at least 3 independent experiments. ${ }^{*} P<0.05$, paired Student's $t$ test.

ologic function of the transduced cells, we first investigated NK-92 and CD16.NK-92 cell ADCC. Although both cell lines killed K562 similarly (Figure 5A), CD16.NK-92 cells demonstrated increased activity against Raji cells in the presence of anti$\mathrm{CD} 20 \mathrm{mAb}$ (rituximab; Figure 5, B and C). In consideration of a role for CD16 in the NK-92 cells in cytotoxicity independent of its function as an Fc receptor, we evaluated cytolytic activity against the mel1106 melanoma cell line. The mel1106 cell line has been previously described as resistant to cytotoxicity by in vitro-expanded CD16- NK cell clones, but sensitive to CD16 ${ }^{+}$ human NK cell clones (22). Since this previous observation could have been a feature of NK cell maturation, the stable NK-92 and CD16.NK-92 cell lines allowed us to directly evaluate CD16 in mel1106 killing. In repeated independent experiments, mel1106 cells were killed better by CD16.NK-92 than by NK-92 parental cells (Figure 5D). To exclude the possibility that ADCC against mel1106 cells had been inadvertently triggered by bovine antibodies in culture media, the same assays were performed with target and effector cells prepared and assayed in serum-free media. Even under serum-free conditions, percent lysis of the mel1106 target cells mediated by CD16.NK-92 cells was higher than that by NK-92 cells (Figure 5E). 

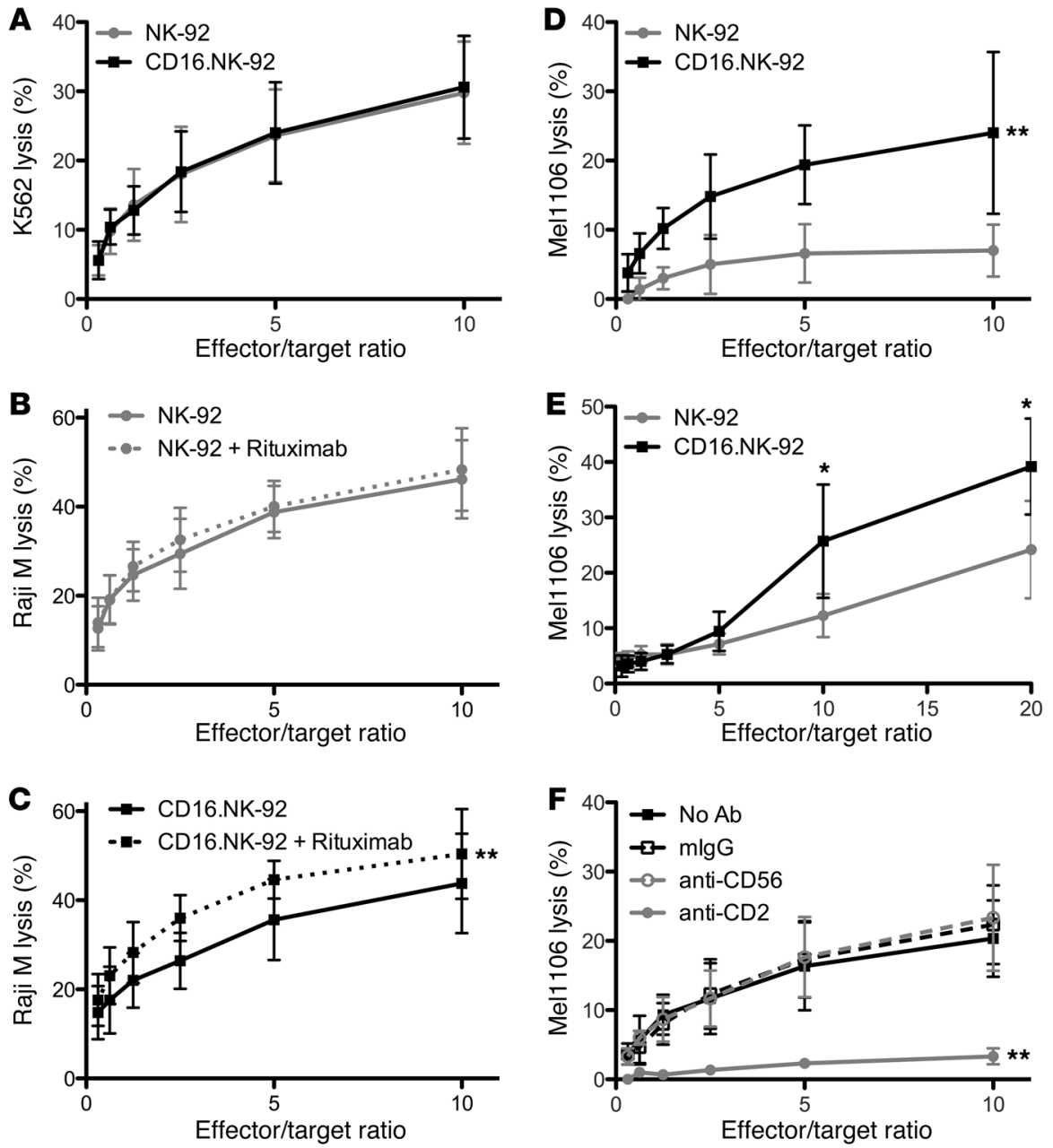

\section{Figure 5}

Functional activity of NK-92 cell lines. Cytotoxic activity was assessed using 4-hour ${ }^{51} \mathrm{Cr}$-release assays. (A) NK-92 or CD16. NK-92 against K562 target cells ( $n=5$ independent experiments). ADCC assays against Raji cells using added rituximab for (B) NK-92 and (C) CD16.NK-92 cells, in which only the latter demonstrated activity ( $n=4$ independent experiments). Spontaneous cytotoxicity against mel1106 cells in the presence (D) and absence (E) of fetal calf serum in NK-92 and CD16.NK-92 cells ( $n=5$ independent assays). (F) Addition of anti-CD2 blocking $m A b$, but not anti-CD56 or nonspecific mlgG, abrogated CD16.NK-92 cytotoxicity against mel1106 cells $(n=3$ independent assays). ${ }^{*} P<0.05$, paired Student's $t$ test (comparisons of individual points); ${ }^{* *} P<0.05$, Wilcoxon signed-rank test (comparisons across independent assays).
To determine whether increased CD2 surface expression on CD16.NK-92 cells could be of potential functional significance, we evaluated the role of CD2 in mel1106 killing. Cytotoxicity was measured in the presence of a blocking anti-CD2 $\mathrm{mAb}$, nonspecific murine IgG (mIgG; isotype control), or a mAb against CD56 (specificity control). No effect on mel1106 cytotoxicity was observed in the presence of $\mathrm{mIgG}$ or anti-CD56, whereas the anti-CD2 antibody significantly decreased killing of mel1106 cells by the CD16. NK-92 cell line (Figure 5F). Thus, CD16 - and, potentially, the associated increased CD2 surface expression - contributed to NK cell-mediated spontaneous cytotoxicity against mel1106 cells. This presented the opportunity to further study CD16.NK-92 cells and pursue a mechanism by which CD16 imparts NK cell spontaneous cytolytic function.

CD16 localizes to the lytic NK cell immunological synapse. Since CD16 expression leads to increased CD2 levels, we asked whether increased target cell killing might simply result from an increased ability to form conjugates with target cells. This seemed plausible, given known roles for CD2 in adhesion (23) and the integral initial role for adhesion in lytic synapse formation (4). Thus, we performed a FACS-based conjugation assay using fluorescent membrane dye-labeled K562 or mel1106 target cells with differentially labeled NK-92 or CD16.NK-92 effector cells. The percentage of conjugates formed over 4 hours with K562 or mel1106 was similar for both NK cell lines, and the presence of CD16 did not lead to significant differences at any individual time point or over the entire time course (Figure 6A). Thus, the presence of CD16 did not affect the efficiency or rate of conjugate formation with either target cell.

After conjugate formation, a subsequent major step in the lytic immunological synapse is aggregation of activating receptors (4). We therefore sought to determine whether CD16 was localized at the synapse formed between CD16.NK-92 and mel1106 cells, using confocal microscopy to identify synaptic accumulation throughout 3 dimensions (Figure 6B). Quantitative analysis of CD16 accumulation was performed by comparing the volume and fluorescence intensity within identically sized $3 \mathrm{D}$ regions at the synapse of conjugated effector cells with those of randomly selected regions on the cell surface of unconjugated cells, as previously described (24). Across multiple independently assessed cells, the level of synaptic CD16 was greater than that at the surface in unconjugated cells (Figure 6C), demonstrating net synaptic accumulation. Thus, CD16 accumulated at the synapse formed with mel1106 target cells for which CD16 is required for lysis.

Expression of CD16 L66H in the NK-92 cell line recapitulates the patient phenotype. To connect the phenotype in our patients and effects of CD16 on cytotoxicity, we stably expressed the CD16 L66H protein in the NK-92 cell line via retroviral transduction to create the CD16. L66H.NK-92 cell line. We confirmed mutant protein expression by 
A
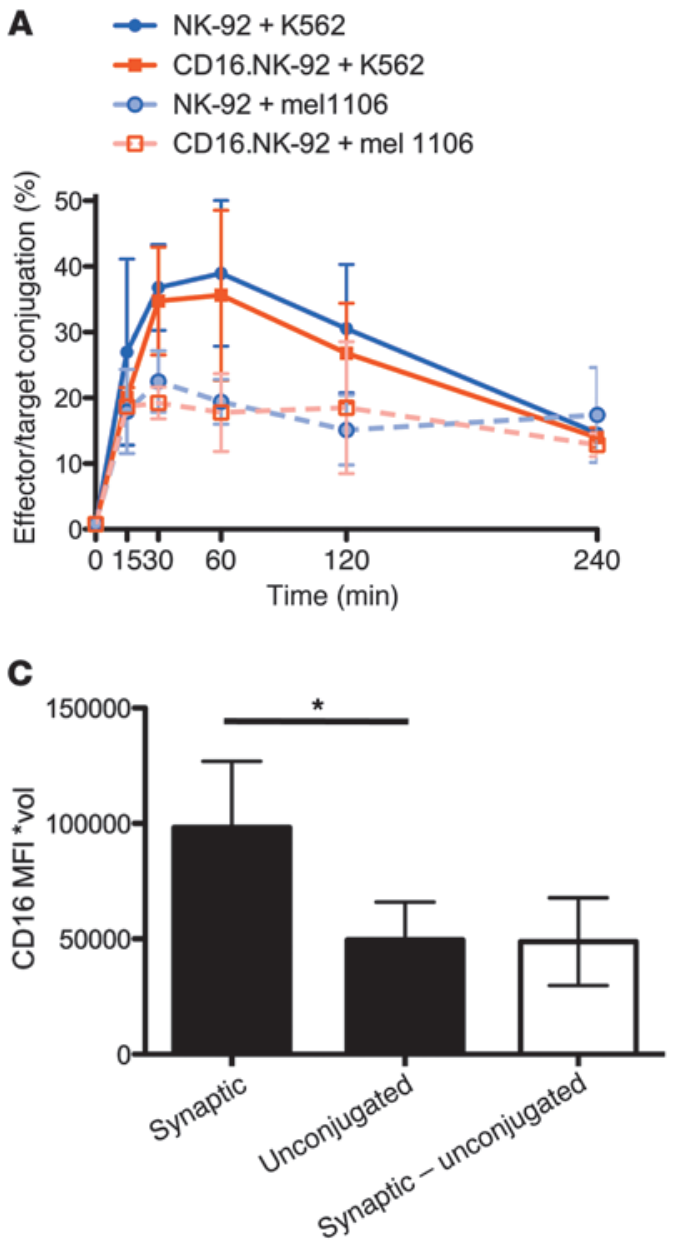

B
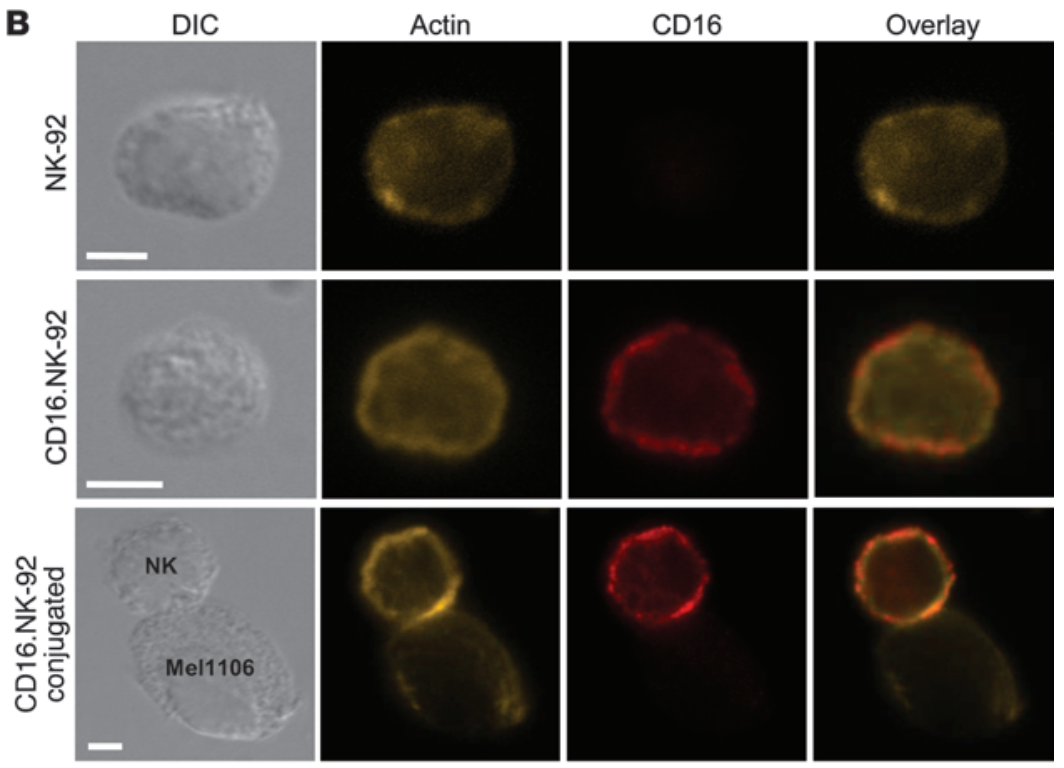

\section{Figure 6}

CD16 does not improve conjugate formation, but accumulates at the mel1106:NK cell synapse. (A) Conjugation assay, expressed as percent NK cells conjugated to target cells over 4 hours ( $n=3$ independent experiments). No significance was identified using Wilcoxon signed-rank test (independent assays) or Student's $t$ test (individual points). (B) Representative fixed-cell images of actin (yellow) or CD16 (red) in unconjugated NK-92 cells, unconjugated CD16.NK-92 cells, and CD16.NK-92 cells conjugated with mel1106 cells. Scale bars: 5 um. 5 conjugates or control cells were analyzed from each of 3 independent experiments ( $n=15$ per condition). (C) CD16 quantitation (mean \pm SD) at the mel1106:NK cell synapse compared with unconjugated effectors, using regions of equal size ( $n=15$ per condition). ${ }^{*} P<0.05$, paired Student's $t$ test. Subtraction of unconjugated CD16 values from CD16 accumulated at the immune synapse demonstrated a mean positive value.

FACS using the $3 \mathrm{G} 8$ and B73.1 mAbs. CD16.L66H.NK-92 cells were only recognized by $3 \mathrm{G} 8$ (Figure 7A), which confirmed that the point mutation in CD16 was directly responsible for the loss of the B73.1 epitope, as had been inferred from the patient cells.

The ability of NK-92, CD16.NK-92, and CD16.L66H.NK-92 cell lines to to kill K562 target cells did not differ (Figure 7B). In contrast, whereas the CD16.NK-92 cell line robustly killed mel1106 target cells, the CD16.L66H.NK-92 cell line was significantly less effective and similar to that of the parental NK-92 cell line (Figure $7 \mathrm{C})$. Thus, the $\mathrm{L} 66 \mathrm{H}$ variation altered CD16 to an extent that it disrupted its contribution to spontaneous killing.

We next asked whether, in this model system, CD16 L66H mutant expression affected surface CD2 levels relative to NK cells expressing wild-type CD16. FACS analysis demonstrated significantly more CD2 on the surface of CD16.NK-92 cells than on either NK-92 or CD16.L66H.NK-92 cells (Figure 7D), which indicates that wild-type CD16 contributes to overall expression of CD2 on the NK cell surface. This was confirmed by Western blot analysis of whole-cell lysates (Figure 7E), which suggests that the overall stability of CD2 in the presence of wild-type, but not mutant, CD16 was improved. These analyses also indicate that the increased amount of CD2 in the CD16.NK-92 cells was not a feature of retroviral transduction, as the level was increased compared with that in CD16.L66H.NK-92 cells.

Normal, but not mutant, CD16 associates with CD2 and provides access to signaling proteins. Since CD2 is known to accumulate at the NK cell lytic synapse $(25,26)$, and wild-type CD16 correlated with increased surface CD2 in NK-92 and ex vivo human NK cells, we asked whether there might be an association between CD16 and CD2 on NK cells dependent upon the region of CD16 affected by the mutation. We first explored this possibility using confocal microscopy to measure the colocalization of CD16 and CD2 on the NK cell surface after activation in conjugates between CD16. NK-92 or CD16.L66H.NK-92 and mel1106 cells. Although CD2 

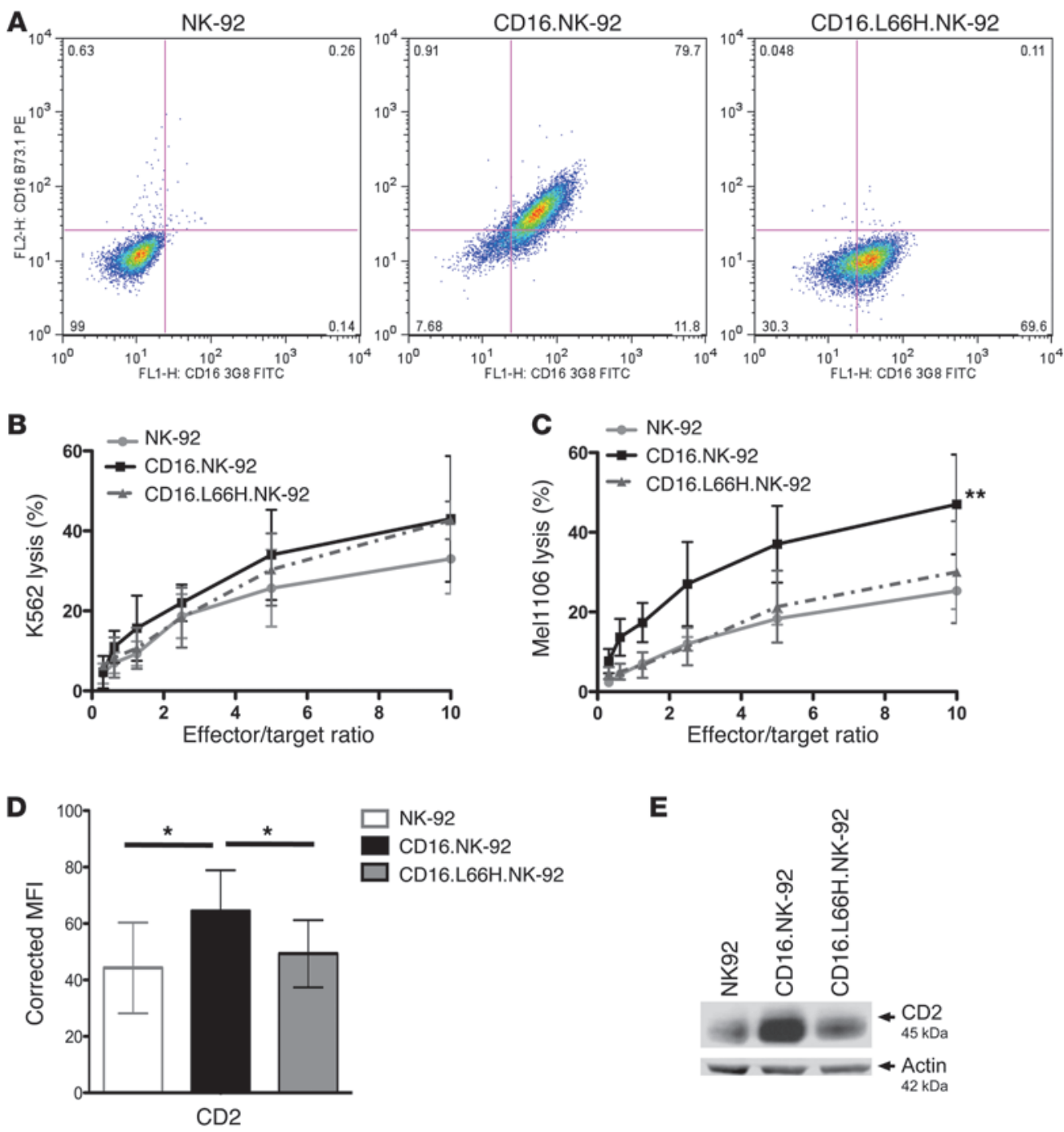

E

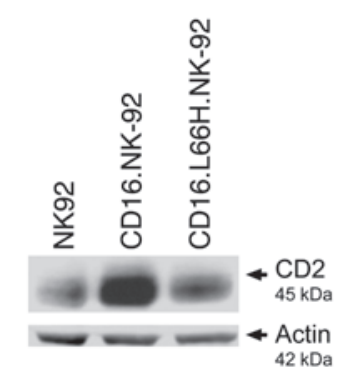

\section{Figure 7}

The CD16.L66H.NK-92 cell line phenotypically and functionally recapitulates patient NK cells. (A) FACS analysis of NK-92, CD16.NK-92, and CD16.L66H. NK-92 cell lines using CD16 mAbs, 3G8, and B73.1 (representative of $n=5$ ). (B) Spontaneous cytotoxicity against K562 target cells $(n=3$ independent experiments; no significant differences). (C) Spontaneous cytotoxicity against mel1106 cells. ${ }^{* *} P<0.05$, Wilcoxon signed-rank test ( $n=3$ independent experiments). (D) FACS analysis of surface CD2. Corrected MFI (relative to isotype control) was significantly greater in CD16.NK-92 cells. ${ }^{\star} P<0.05$, paired Student's $t$ test $(n=5$ individual experiments). (E) Western blot analysis of CD2 expression in whole cell lysates. Actin was used as a loading control on the same membrane following stripping and reprobing (representative of 5 independent experiments). accumulated at the synapse in all of the effector cell lines, only wild-type, not L66H mutant, CD16 also accumulated (Figure 8A). Quantitative analysis demonstrated that CD16 preferentially colocalized with CD2 at the immune synapse compared with CD56, used as a nontriggering receptor control. Furthermore, wild-type CD16 showed significantly greater colocalization with CD2 than did mutant CD16 L66H (Figure 8B).

Since wild-type, but not L66H mutant, CD16 and CD2 colocalized at the synapse, we postulated a specific association and interaction between the 2 molecules, which we studied further using CD2 immunoprecipitation. Compared with the proteins precipitated using an isotype control, CD2 immunoprecipitates from CD16.NK-92, but not NK-92, cell lysates demonstrated the presence of CD16 (Figure 8C). Thus, CD16 could interact with CD2 in NK cells. Importantly, this interaction was not identified in NK cells expressing the CD16 L66H protein (Figure 8C), thereby defining the specificity of the interaction between CD16 and CD2 as being dependent on the epitope recognized by monoclonal B73.1, which was mutated in our patients.

Finally, we sought to determine whether the association between CD2 and CD16 was of functional relevance with regard to activation signal generation in NK cells, and how this might be affected by the patient-derived mutant. Although the presence of CD16 in CD16.NK-92 cells extended their target cell range for cytotoxicity (Figure 5), it was not caused by an increased ability to form conjugates. Thus, we hypothesized that the association with CD2 engages CD16-associated signaling and asked whether the TCR $\zeta$ signaling adaptor associated with CD16 is found in the CD16-CD2 complex. TCR $\zeta$ has thus far only been linked to CD2 via functional assays in NK cells $(27,28)$. Upon immunoprecipitation of CD2, TCR $\zeta$ was only identified in precipitates from CD16.NK-92 cells, not parental CD16- NK-92 or CD16.L66H.NK-92 cells (Figure 8C). Thus, the patient-derived mutant CD16 blocked the inclusion of TCR $\zeta$ in the CD2 complex.

Given the association between TCR $\zeta$ and wild-type CD16, we asked whether CD2 ligation could engage CD16 signaling through TCR $\zeta$ and whether it might explain the augmented function relative to cells containing the patient-derived mutation. We triggered CD2 by antibody crosslinking and assayed for TCR $\zeta$ phosphorylation following immunoprecipitation. Crosslinking with anti-CD2 antibody, but not control anti-CD56 antibody, induced TCR $\zeta$ phosphorylation in CD16.NK-92 cells. In contrast, the increase in TCR $\zeta$ phosphorylation was substantially reduced when CD2 was crosslinked in the CD16.L66H.NK-92 cell line 
A
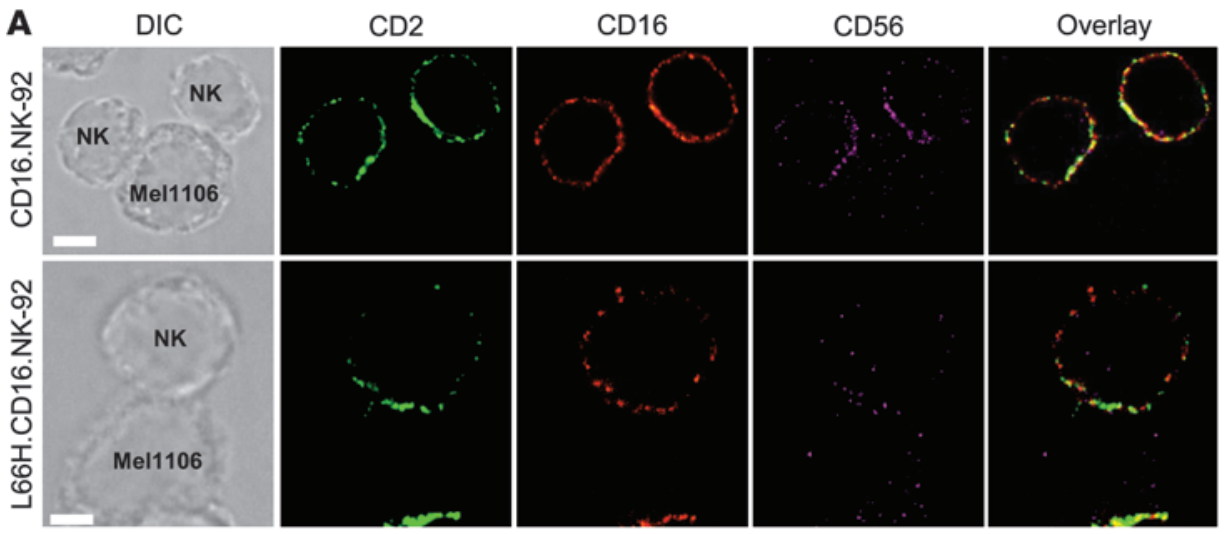

$\mathbf{B}$

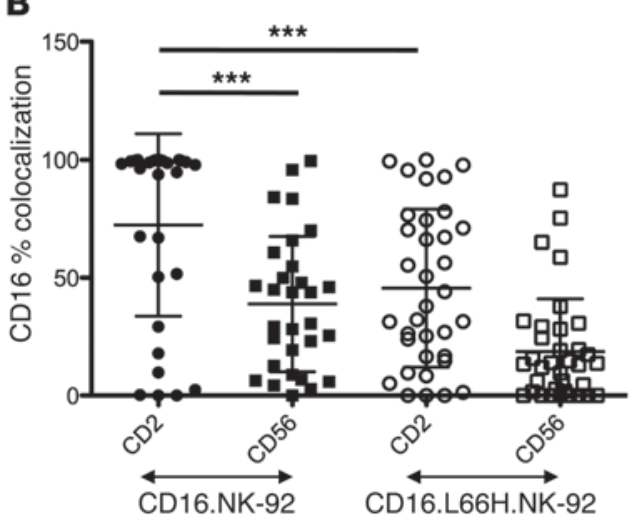

c

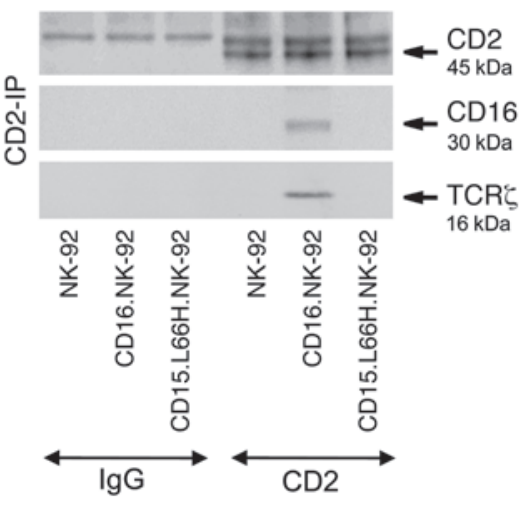

D

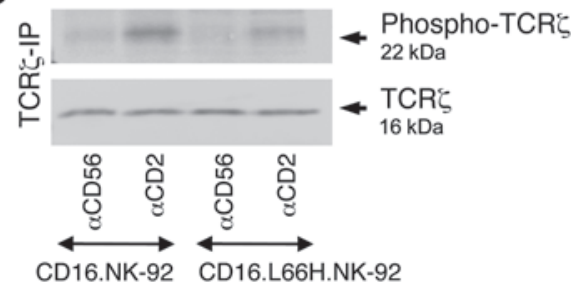

\section{Figure 8}

CD16 associates with CD2 and engages CD16 signaling machinery. (A) Confocal immunofluorescence for CD16 (red) localization relative to CD2 (green) and CD56 (purple) at the immune synapse in CD16. NK-92 and CD16.L66H.NK-92 conjugated with mel1106 cells. Images are representative of $n=30$ across 3 independent experiments. Scale bars: $5 \mu \mathrm{m}$. (B) Quantitative analysis of percent CD16 colocalized with CD2 (circles) or CD56 (squares) at the immune synapse of CD16. NK-92:mel1106 (filled symbols) or CD16.L66H.NK-92:mel1106 (open symbols). Each point represents percent colocalization from a single conjugate; horizontal lines denote mean; error bars denote SD. ${ }^{* *} P<0.0001$, unpaired Student's $t$ test; $n=30$. (C) CD2 immunoprecipitation from the indicated cell lines and Western blot analysis for CD2, CD16, and TCR $\zeta$, all using the same membrane after stripping and reprobing (representative of $n=5$ ). (D) Western blot analysis of phosphorylated TCR $\zeta$ in TCR $\zeta$ immunoprecipitates from CD16. NK-92 and CD16.L66H.NK-92 cells activated with either antiCD2 or anti-CD56 Ab and then goat anti-mouse IgG. Total TCR $\zeta$ is shown as a loading control. Representative of 3 experiments.
(Figure 8D). Thus, CD2 interacted with CD16 and induced activation signaling through the nonligated CD16 receptor complex, thereby increasing signal strength. Furthermore, this costimulatory pathway was rendered defective by the CD16 L66H mutation, which suggests direct importance of this CD16 region and that the loss of this mechanism underlies the functional NK cell deficiency and associated clinical phenotype found in patients affected by this abnormality.

\section{Discussion}

NK cells are essential for host defense against certain infectious diseases and serve important roles in tumor cell surveillance (29). Patients with NK cell deficiencies are susceptible to severe viral infections, especially those caused by herpesviruses (14). While some patients with NK cell deficiencies have difficulty producing NK cells, others maintain NK cells that are functionally impaired. A previously reported mutation of CD16 resulted in a functional NK cell deficiency in a single patient with susceptibility to herpesvirus. In particular, homozygous CD16 L66H mutation was associated with decreased NK cell cytotoxicity, but normal ADCC (16). Patients with pure antibody deficiency
(X-linked agammaglobulinemia) do not have increased susceptibility to herpesviruses, which makes it unlikely that ADCC and CD16 binding to IgG is responsible for the phenotype observed in the $\mathrm{CD} 16 \mathrm{~L} 66 \mathrm{H}$ patients (30). This raised the possibility that CD16 might have a role in NK cell cytotoxicity independent from its function in ADCC. To better define this rare, but naturally occurring, human mutation of CD16 as an immunodeficiency disorder, and to consider a mechanistic role for CD16 in NK cell cytotoxicity, we identified and characterized a new and unrelated patient homozygous for the same $\mathrm{L} 66 \mathrm{H}$ mutation.

Patient 1 was identified among a cohort having unusual manifestations of herpesvirus infections through an assay screening for the absence of the CD16 epitope recognized by mAb B73.1, as had been previously defined in patient 2 . While we used the loss of the B73.1 epitope to initially identify patient 1 , a recent report defined the loss of this epitope in a relatively large number of patients with recurrent infection, none of whom carried homozygous mutations of CD16 within the region encoding the epitope (31). Given the similarity between the genes encoding CD16A and CD16B, which is not expressed on NK cells (32), proof of specificity for CD16A must be sought during genotyping assays. Nonetheless, the use of a flow 
cytometry-based assay to identify patients with a CD16 L66H mutation can be used in screening, but gene sequencing is needed for confirmation. Because 167 patients with suggestive clinical phenotypes were evaluated in that study (31), it follows that homozygosity for the $\mathrm{L} 66 \mathrm{H}$ variant is rare, even among highly preselected populations. Our experience confirmed that finding, since we only identified a single subject within our cohort of 30 even more highly preselected patients.

In both patient 1 and patient 2 , spontaneous cytotoxicity was impaired, but ADCC was preserved. This suggests that CD16 - specifically, the distal Ig domain containing L66 - can facilitate spontaneous cytotoxicity independently of function as an Fc receptor. An hypothesis proposing CD16 as a costimulatory receptor for spontaneous NK cell cytotoxicity has been raised previously, although the mechanistic basis remained unclear (22). Given our patient-related observation, we pursued a role for CD16, and specifically the distal Ig domain, in NK cell cytotoxicity using both patient NK cells and an NK-92 cell line model. In the latter, the only differences were the stable expression of CD16 and CD16 L66H, or the lack of CD16 expression. The most consistent phenotypic finding was the decreased expression of CD2 in patient NK cells or in CD16-deficient and CD16 L66H-expressing NK-92 cells. Thus we, hypothesized a role for CD16 in spontaneous cytotoxicity via an interaction with CD2 that depends on the integrity of L66 within the membrane distal Ig domain.

CD2 is a known NK cell activation receptor, which mediates adhesion and signal transduction $(25,33)$. CD2 can aggregate at the NK cell immunological synapse $(25,26)$, and crosslinking of CD2 on the surface of NK cells induces tyrosine kinase signal transduction and lytic activity (34). While the role of CD2 in spontaneous NK cell cytotoxicity has been evaluated, to our knowledge, specific interaction of $\mathrm{CD} 2$ with other activating receptors (such as CD16) on the NK cell surface has not been previously addressed.

To obtain mechanistic insight into the role of CD16 in spontaneous cytotoxicity of NK cells, the mel1106 target cell was considered, since lysis of this target cell was previously defined as being only possible by $\mathrm{CD} 16^{+} \mathrm{NK}$ cell clones (22). In this light, NK cells are well known to have a function in defense against melanoma, as the CD56 ${ }^{\text {dim }} \mathrm{CD} 16^{+}$subset of NK cells preferentially targets metastatic melanoma in lymph nodes compared with CD56 $6^{\text {bright }}$ CD $16^{-}$cells (35). Additionally, in melanoma patients treated with IL-2, CD16 ${ }^{+}$ NK cells demonstrate increased cytotoxicity over NK cells not expressing CD16 (36). While this may represent an inherent property of CD $16^{+}$compared with $\mathrm{CD} 16^{-} \mathrm{NK}$ cells, our results suggest a potential role for CD2: targeting NK cells to melanoma. A survey of melanoma cell lines in the European Searchable Tumour Cell Line and Data Bank identified all of the cataloged melanoma cell lines as expressing CD58, a cell adhesion molecule and natural ligand for CD2 (37). We confirmed the presence of CD58 on the mel1106 cell line used in our experiments (data not shown). Moreover, in melanoma cell lines susceptible to NK cell lysis, anti-CD58 mAb inhibited $15 \%-45 \%$ of NK cytotoxicity (38). In agreement with this, we observed that anti-CD2 mAb significantly reduced CD16.NK-92 cytotoxicity against the mel1106 cell line; thus, the CD2/CD58 interaction likely contributes to NK cell activation and targeting toward melanoma cells. The host factors that define successful NK cell-mediated spontaneous cytotoxicity against melanoma are clearly more complex, but our present findings suggest a rationale for considering a functional association between CD2 and CD16.

Given that CD16 is an IgG Fc receptor, it is difficult to exclude a role for $\operatorname{IgG}$ (even from a different species) in the activity against mel1106 cells. In the original study, NK cell lines were cultured in media containing human serum, which could have activated CD16 signaling prior to target cell exposure. In the present study, we were able to confirm the utility of CD16 in lytic activity against mel1106 cells, even under serum-free conditions. In addition, the presence of CD16 did not impart a greater ability for NK cells to conjugate to target cells, which further suggested it was unlikely that there was target cell-bound IgG Fc ligand present.

In support of a role for CD16 in mel1106 cell killing, however, CD16 clustered at the immunological synapse formed with mel1106 cells and coimmunoprecipitated with CD2. This suggested that CD2 could interact with CD16, thus involving it in the lytic process, regardless of the presence of an IgG Fc ligand. The patient results and predicted mutant CD16 structure suggest that the mechanism of this interaction involves the membrane distal, non-Fc-binding Ig domain of CD16. This conclusion was further supported by the CD16.L66H.NK-92 cell line, which contains the exact patient mutation in the distal domain. This cell line demonstrated that the integrity of the B73.1 epitope and L66 were necessary for interaction between CD16 and CD2, as the CD16 L66H protein did not accumulate at the immunological synapse or coimmunoprecipitate with CD2. Given that the CD16.L66H.NK-92 cell line had a diminished capacity to kill mel1106 cells, we propose that CD16 functions in spontaneous NK cell cytotoxicity through an interaction with CD2 via the non-Fc-binding distal domain of CD16.

Despite the wealth of information regarding CD16, the function of its distal Ig-like domain remains unclear, and interactions with other proteins through this domain of CD16 have not been previously described. Chimeric proteins in which either the distal Ig domain or the membrane-proximal domain had been replaced with Ig domains of ICAM-1 demonstrated that the distal domain was necessary for surface expression and binding of anti-CD16 mAbs to the respective domain (7). This suggests a potential function of the first, distal Ig domain of CD16 in the structural integrity and stability of the protein as a whole. Although we are unaware of other known protein interactions at the distal domain of CD16, protein-ligand interactions have been described for the distal domain of other NK cell receptors of the Ig superfamily. For example, the ligand-binding site of CD2 is on the distal Ig-like domain (39). However, we are only aware of these interactions occurring in trans and not cis orientations. In this light, it is unclear whether the interaction of CD16 with CD2 is mediated by a direct physical interaction through the distal Ig-like domain of CD16, or whether the distal domain is involved in an interaction with another protein or complex that bridges the 2 transmembrane proteins. In either case, to our knowledge, our finding would represent the first identified cis interaction specific to only the far distal domain of an Ig superfamily receptor. Alternatively, alteration of the distal Ig-like domain of CD16 could have a greater effect on the overall structure of the protein than our predicted $\mathrm{L} 66 \mathrm{H} 3 \mathrm{D}$ model, leading to an abnormal interaction at some other part of the molecule.

Given that CD16 is known to have substantial potency in triggering ADCC, the interaction of ligated CD2 with CD16 could be of potential benefit in NK cell activation during spontaneous cytotoxicity. The CD16 signaling machinery functions independently of many other activation receptors on NK cells and can operate effectively without a coactivation receptor to trigger cytolytic activity or cytokine release (40). Thus, through an interaction with CD16 via its distal Ig domain, CD2 could gain access to signaling apparatus capable of conveying a strong activation signal.

When we evaluated the involvement of CD16 signaling downstream of CD2 activation, we identified phosphorylation of the 
CD16-associated signaling adaptor TCR $\zeta$. Although TCR $\zeta$ is a known intermediate of CD16 signal transduction, it has also been implicated in CD2 signaling. In both NK cells and T cells, CD2 signal transduction is functionally dependent upon TCR $\zeta$, of which CD2 ligation can promote phosphorylation $(27,28)$. Additionally, in NK cells, it was previously found that the presence of CD16 was required for phosphorylation of TCR $\zeta$ following CD2 activation (27), yet the physical basis of association between CD2 and TCR $\zeta$ remained unclear. Our data demonstrated that the membrane-distal Ig-like domain of CD16 was likely to enable a unique cis interaction with CD2, which could be used by CD2 to gain access to TCR $\zeta$ and promote costimulation for NK cell spontaneous cytotoxicity.

We here defined CD16, an Fc receptor, as functioning in NK cell spontaneous cytotoxicity, independently of antibody binding and via its distal Ig-like domain. Through analysis of a rare homozygous patient mutation, we concluded that CD16 participates in an interaction with the coactivation receptor $\mathrm{CD} 2$, which activates the CD16 signaling machinery following CD2 ligation. Furthermore, the interaction between CD16 and CD2 may occur via a unique cis protein interaction using the non-Fc-binding, distal Ig domain of CD16. This interaction was defective in a human immunodeficiency resulting from a CD16 abnormality and, given the associated viral susceptibility, is likely to represent the underlying immunological mechanism, thus attributing critical importance to this specific NK cell function and NK cell-mediated defense.

\section{Methods}

Patient screening and selection. During a 5-year period, 30 patients with recurrent herpesviral susceptibility were screened for NK cell phenotypic and functional deficiencies. Presence of CD16 epitopes (dual positivity, see below) and presence of NK cell cytotoxicity was assayed. For patients lacking the CD16 B73.1 $\mathrm{mAb}$ epitope, $\mathrm{CD} 16$ was sequenced as described previously (16).

Patients. 2 patients with abnormal CD16 dual positivity were studied. Patient 1 was a 14-year-old male with recurrent lymph node EBV-driven Castleman's disease beginning at 10 years of age, who developed papillomavirus of the hands and feet at 14 years of age (Supplemental Figure 1). Given his susceptibility to recurrent EBV and HPV, evaluation of immunity was performed and demonstrated normal absolute numbers of neutrophils, major T cell subsets, and B cells. Mitogen and Candida antigen-induced lymphocyte proliferation was normal, as were Ig isotype quantitative levels and specific IgG titers against tetanus, diphtheria, and pneumococcus. EBV Viral Capsid (VCA) IgG titer was 1:320. Patient 2 is a 22-year-old female previously reported at age 5 (16).

Human NK cell preparation and NK and target cell lines. PBMCs were isolated from heparinized whole blood using Ficoll Hypaque (Amersham). NK-92, an immortalized human NK cell line lacking endogenous CD16, was stably transduced with a CD16 retroviral expression construct to create CD16.NK-92 cells, as described previously (21), and a CD16 retroviral construct encoding the $\mathrm{L} 66 \mathrm{H}$ patient mutation was used to create the CD16.L66H.NK-92 cell line.

K562 erythroleukemia and Raji B cell lines were used as target cells for NK cell cytotoxicity and ADCC, respectively. Additionally, mel1106 melanoma cells were used as target cells.

CD16 dual-positivity assay and NK phenotyping. Patient whole blood was evaluated for $\mathrm{CD}^{-} \mathrm{CD} 56^{+} \mathrm{NK}$ cells in a $\mathrm{CD} 45^{+}$leukocyte gate for the presence of CD16 using 2 distinct mAbs (B73.1 and 3G8; BD Biosciences). A normal result was defined as NK cells that were dually positive. Extended NK cell phenotyping was performed using PBMCs and mAb sets (Supplemental Table 1) as described previously (41). Nonspecific staining was determined using isotypematched control antibodies, and background-corrected MFI was reported.
Cytotoxicity assays. NK-92, CD16.NK-92, and CD16.L66H.NK-92 cells and PBMCs were used as effectors against ${ }^{51} \mathrm{Cr}$-labeled K562, Raji, and mel1106 cells in 4-hour ${ }^{51} \mathrm{Cr}$-release assays, and lytic units were calculated as described previously (42). Serum-free assays were performed using target cells that had been preincubated in serum-free medium at room temperature for at least 30 minutes. Where specified, CD16.NK-92 cells were preincubated with $\mathrm{mIgG}$, anti-CD56, or anti-CD2 $\mathrm{mAb}(20 \mu \mathrm{g} / \mathrm{ml})$, and cytotoxicity was assayed in the presence of the antibody.

$3 \mathrm{D}$ modeling. The predicted 3D model of CD16 $\mathrm{L} 66 \mathrm{H}$ protein was generated using 3D-Jigsaw $(43,44)$, based on the CD16 1E4J protein structure (19), and modeled in Swiss PDB Viewer.

Conjugation assays. NK-92 effector cells were labeled with 0.625M CFSE at $37^{\circ} \mathrm{C}$ for 20 minutes, then washed and incubated in prewarmed RPMI- 1640 medium containing $10 \%$ heat-inactivated FCS at $37^{\circ} \mathrm{C}$ for 30 minutes. Target cells were labeled with 1.25-2.5 nM pKH26 (Sigma-Aldrich) for 5 minutes at $20^{\circ} \mathrm{C}$ prior to washing. Effectors and targets were combined 2:1, and $2 \%$ paraformaldehyde was added to aliquots at $0,15,30,60,120$, and 240 minutes to fix for FACS analysis. Conjugates were defined as $\mathrm{CFSE}^{+} \mathrm{pKH} 26^{+}$, unconjugated effectors as $\mathrm{CFSE}^{+} \mathrm{pKH} 26^{-}$, and unconjugated targets as CFSE-pKH $26^{+}$. The percentage of NK cells conjugated was determined as the number of $\mathrm{CFSE}^{+} \mathrm{pKH} 26^{+}$relative to the total $\mathrm{CFSE}^{+}$events.

Confocal microscopy. NK-92 and mel1106 cells were mixed 2:1 and adhered to poly-L-lysine-coated slides at $37^{\circ} \mathrm{C}$ for 30 minutes as described previously (26). Slides were blocked with $1 \%$ BSA for 20 minutes, washed, and incubated with $20 \mu \mathrm{g} / \mathrm{ml}$ anti-CD163G8 mAb for 40 minutes at $4^{\circ} \mathrm{C}$, followed by Alexa Fluor 568 goat anti-mouse IgG (Invitrogen). Unoccupied anti-mouse IgG antibody was then blocked with $\mathrm{mIgG}$ followed by $5 \mu \mathrm{g} / \mathrm{ml}$ mouse antihuman CD2-biotin (BioLegend), followed by streptavidin-Alexa Fluor 647 (Invitrogen) and Brilliant Violet anti-CD56 (BioLegend). Where specified, cells were permeabilized as described previously (26) and incubated with phalloidin 647 (Invitrogen). Slides were mounted with Pro-long anti-fade (Invitrogen). Imaging was performed using spinning disc confocal microscopes (Olympus and Zeiss) with $\times 601.49 \mathrm{NA}$ or $\times 631.49 \mathrm{NA}$ objective. Images were captured and analyzed throughout the $z$ axis at $0.5-\mu \mathrm{m}$ intervals using Volocity (Perkin-Elmer). Quantitation of fluorescent accumulation at the immune synapse was calculated as described previously (24).

Immunoprecipitation. $1 \times 10^{7}$ cells were lysed in $0.1 \% \mathrm{NP}-40,25 \mathrm{mM}$ Tris$\mathrm{HCl}$, and $150 \mathrm{mM} \mathrm{NaCl}$ with protease inhibitor (Sigma-Aldrich) on ice for 30 minutes. Since cell lines expressed unequal amounts of CD2, Western blot analysis of each lysate was used for volume normalization, after which immunoprecipitation was performed. Lysates were precleared with $50 \mu \mathrm{l}$ unlabeled rProtein $\mathrm{G}$ agarose beads (Invitrogen), then incubated at $4^{\circ} \mathrm{C}$ preloaded with anti-CD2 TS2/18.1 mAb or with mouse IgG mAb MOPC21 (BD) rProtein G agarose beads. Beads were then washed and boiled in NuPAGE sample buffer (Invitrogen), and supernatants were run under reducing conditions in 4\%-12\% Bis-Tris NuPAGE gels (Invitrogen).

For TCR $\zeta$ immunoprecipitation, cells were preactivated with either anti$\mathrm{CD} 2$ or anti-CD56 mAbs cross-linked by goat anti-mouse IgG, as described previously (28), in serum-free media to avoid CD16 triggering. Lysates were precleared and and immunoprecipitated using beads preloaded with antiTCRל (Santa Cruz).

Statistics. For data consisting of multiple repeats, mean \pm SD is shown. Individual, repeated experimental data points were analyzed for significance using 2-tailed paired and unpaired Student's $t$ test for paired and unpaired sets, respectively. For continuous data sets, the nonparametric Wilcoxon signed-rank test was performed. Differences were considered significant for $P$ values less than 0.05 .

Study approval. All patient evaluations of NK cell deficiency were performed with informed consent using a form and protocol approved by the Children's Hospital of Philadelphia and Baylor College of Medicine IRBs. 


\section{Acknowledgments}

The authors thank Michelle Paessler for histopathology. This work was supported by NIH grant R01-AI067946 and a Jeffrey Modell Foundation center grant (to J.S. Orange) and by NIH grant R01CA083859 and a Pennsylvania Department of Health Tobacco CURE grant (to K.S. Campbell).
Received for publication May 17, 2012, and accepted in revised form July 26, 2012.

Address correspondence to: Jordan Orange, 1102 Bates St., FC330, Houston, Texas 77030-2399, USA. Phone: 832.824.1319; Fax: 832.825.2112; E-mail: orange@bcm.edu.
1. Bryceson YT, et al. Molecular mechanisms of natural killer cell activation. J Innate Immun. 2011;3(3):216-226.

2. Lanier LL, Phillips JH, Testi R. Membrane anchoring and spontaneous release of CD16 (FcR III) by natural killer cells and granulocytes. Eur J Immunol. 1989;19(4):775-778.

3. Vivier E, Tomasello E, Baratin M, Walzer T, Ugolini S. Functions of natural killer cells. Nat Immunol. 2008;9(5):503-510.

4. Orange JS. Formation and function of the lytic NK-cell immunological synapse. Nat Rev Immunol. 2008;8(9):713-725.

5. Lanier LL, Cwirla S, Yu G, Testi R, Phillips JH. Membrane anchoring of a human IgG Fc receptor (CD16) determined by a single amino acid. Science. 1989;246(4937):1611-1613

6. Lanier L, Ruitenberg J, Phillips J. Functional and biochemical analysis of CD16 antigen on natural killer cells and granulocytes. J Immunol. 1988;141(10):3478-3485.

7. Hibbs M, Tolvanen M, Carpen O. Membrane-proximal Ig-like domain of Fc gamma RIII (CD16) contains residues critical for ligand binding. J Immunol. 1994;152(9):4466-4474.

8. Tamm A, Schmidt R. The binding epitopes of human CD16 (Fc gamma RIII) monoclonal antibodies. Implications for ligand binding. I Immunol. 1996;157(4):1576-1581.

9. Lanier LL, Yu G, Phillips JH. Analysis of Fc gamma RIII (CD16) membrane expression and association with CD3 zeta and Fc epsilon RI-gamma by site-directed mutation. J Immunol. 1991;146(5):1571-1576.

10. Perussia B, Trinchieri G. Antibody 3G8, specific for the human neutrophil Fc receptor, reacts with natural killer cells. J Immunol. 1984;132(3):1410-1415.

11. O'Shea JJ, Weissman AM, Kennedy IC, Ortaldo JR Engagement of the natural killer cell IgG Fc receptor results in tyrosine phosphorylation of the zeta chain. Proc Natl Acad Sci U S A. 1991;88(2):350-354.

12. Perussia $B$, et al. The Fc receptor for IgG on human natural killer cells: phenotypic, functional, and comparative studies with monoclonal antibodies. J Immunol. 1984;133(1):180-189.

13. Orange JS. Human natural killer cell deficiencies and susceptibility to infection. Microbes Infect. 2002;4(15):1545-1558.

14. Orange JS. Human natural killer cell deficiencies. Curr Opin Allergy Clin Immunol. 2006;6(6):399-409.

15. Bonilla FA, et al. Practice parameter for the diagnosis and management of primary immunodeficiency. Ann Allergy Asthma Immunol. 2005;94(5 suppl 1):S1-S63.

16. Jawahar S, Moody C, Chan M, Finberg R, Geha R, Chatila T. Natural killer (NK) cell deficiency associated with an epitope-deficient $\mathrm{Fc}$ receptor type IIIA (CD16-II). Clin Exp Immunol. 1996;103(3):408-413.
17. de Vries E, et al. Identification of an unusual Fc gamma receptor IIIa (CD16) on natural killer cells in a patient with recurrent infections. Blood. 1996;88(8):3022-3027.

18. Comans-Bitter WM, et al. Immunophenotyping of blood lymphocytes in childhoodReference values for lymphocyte subpopulations. J Pediatr. 1997;130(3):388-393.

19. Sondermann P, Huber R, Oosthuizen V, Jacob U. The 3.2-A crystal structure of the human IgG1 Fc fragment-Fc gammaRIII complex. Nature. 2000;406(6793):267-273.

20. Caligiuri MA. Human natural killer cells. Blood. 2008;112(3):461-469.

21. Binyamin L, Alpaugh RK, Hughes TL, Lutz CT, Campbell KS, Weiner LM. Blocking NK cell inhibitory self-recognition promotes antibody-dependent cellular cytotoxicity in a model of anti-lymphoma therapy. J Immunol. 2008;180(9):6392-6401.

22. Mandelboim O, Malik P, Davis DM, Jo CH, Boyson JE, Strominger JL. Human CD16 as a lysis receptor mediating direct natural killer cell cytotoxicity. Proc Natl Acad Sci U S A. 1999;96(10):5640-5644.

23. Wyss DF, et al. Conformation and function of the $\mathrm{N}$-linked glycan in the adhesion domain of human CD2. Science. 1995;269(5228):1273-1278.

24. Banerjee PP, Orange JS. Quantitative measurement of F-actin accumulation at the NK cell immunological synapse. J Immunol Methods. 2010;355(1-2):1-13.

25. Inoue $\mathrm{H}$, et al. Lipid rafts as the signaling scaffold for NK cell activation: tyrosine phosphorylation and association of LAT with phosphatidylinositol 3-kinase and phospholipase C- $\gamma$ following CD2 stimulation. Eur I Immunol. 2002;32(8):2188-2198.

26. Orange JS, Harris KE, Andzelm MM, Valter MM, Geha RS, Strominger JL. The mature activating natural killer cell immunologic synapse is formed in distinct stages. Proc Natl Acad Sci U S A. 2003;100(24):14151-14156

27. Moingeon $\mathrm{P}$, et al. CD3 zeta dependence of the CD2 pathway of activation in $\mathrm{T}$ lymphocytes and natural killer cells. Proc Natl Acad Sci U S A. 1992;89(4):1492-1496.

28. Vivier E, Morin PM, O’Brienm C, Schlossman SF, Anderson P. CD2 is functionally linked to the $\zeta$-natural killer receptor complex. Eur J Immunol. 1991;21(4):1077-1080.

29. Sun JC, Lanier LL. NK cell development, homeostasis and function: parallels with CD8+ T cells. Nat Rev Immunol. 2011;11(10):645-657.

30. Winkelstein JA, et al. X-linked agammaglobulinemia: report on a United States registry of 201 patients. Medicine (Baltimore). 2006;85(4):193-202.

31. Lenart M, et al. The loss of the CD16 B73.1/Leu11c epitope occurring in some primary immunodeficiency diseases is not associated with the Fcgam-
maRIIIa-48L/R/H polymorphism. Int J Mol Med. 2010;26(3):435-442.

32. Ravetch JV, Perussia B. Alternative membrane forms of FC gamma RIII(CD16) on human natural killer cells and neutrophils. Cell type-specific expression of two genes that differ in single nucleotide substitutions. J Exp Med. 1989;170(2):481-497.

33. Timonen T, Gahmberg C, Patarroyo M. Participation of CD11a-c/CD18, CD2 and RGD-binding receptors in endogenous and interleukin-2-stimulated NK activity of CD3-negative large granular lymphocytes. Int J Cancer. 1990;46(6):6.

34. Umehara $\mathrm{H}$, et al. Role for adapter proteins in costimulatory signals of CD2 and IL- 2 on NK cell activation. Mol Immunol. 2002;38(8):587-596.

35. Lakshmikanth T, et al. NCRs and DNAM-1 mediate NK cell recognition and lysis of human and mouse melanoma cell lines in vitro and in vivo. J Clin Invest. 2009;119(5):1251-1263.

36. McKenzie RS, Simms PE, Helfrich BA, Fisher RI, Ellis TM. Identification of a novel CD56- lymphokine-activated killer cell precursor in cancer patients receiving recombinant interleukin 2. Cancer Res. 1992;52(22):6318-6322.

37. Casado J, et al. Expression of adhesion molecules and ligands for activating and costimulatory receptors involved in cell-mediated cytotoxicity in a large panel of human melanoma cell lines. Cancer Immunol Immunother. 2009;58(9):1517-1526.

38. Altomonte M, et al. Differential expression of cell adhesion molecules CD54/CD11a and CD58/CD2 by human melanoma cells and functional role in their interaction with cytotoxic cells. Cancer Res. 1993;53(14):3343-3348.

39. Wang JH, et al. Structure of a heterophilic adhesion complex between the human CD2 and CD58 (LFA-3) counterreceptors. Cell. 1999;97(6):791-803.

40. Bryceson YT, March ME, Ljunggren H-G, Long EO. Synergy among receptors on resting NK cells for the activation of natural cytotoxicity and cytokine secretion. Blood. 2006;107(1):159-166.

41. van Montfrans JM, et al. CD27 deficiency is associated with combined immunodeficiency and persistent symptomatic EBV viremia. J Allergy Clin Immunol. 2012;129(3):787-793 e786.

42. Orange JS, et al. IL-2 induces a WAVE2-dependent pathway for actin reorganization that enables WASp-independent human NK cell function. JClin Invest. 2011;121(4):1535-1548.

43. Bates PA, Kelley LA, MacCallum RM, Sternberg MJE. Enhancement of protein modeling by human intervention in applying the automatic programs 3D-JIGSAW and 3D-PSSM. Proteins. 2001;suppl 5:39-46.

44. Contreras-Moreira B, Bates PA. Domain Fishing: a first step in protein comparative modelling. Bioinformatics. 2002;18(8):1141-1142. 